Review Article

\title{
Systematic Review of Focal Prostate Brachytherapy and the Future Implementation of Image-Guided Prostate HDR Brachytherapy Using MR-Ultrasound Fusion
}

\author{
M. Sean Peach, Daniel M. Trifiletti, and Bruce Libby \\ Department of Radiation Oncology, University of Virginia School of Medicine, Charlottesville, VA 22908, USA \\ Correspondence should be addressed to Bruce Libby; bl8b@hscmail.mcc.virginia.edu
}

Received 16 December 2015; Revised 25 February 2016; Accepted 2 March 2016

Academic Editor: Hendrik Van Poppel

Copyright @ 2016 M. Sean Peach et al. This is an open access article distributed under the Creative Commons Attribution License, which permits unrestricted use, distribution, and reproduction in any medium, provided the original work is properly cited.

Prostate cancer is the most common malignancy found in North American and European men and the second most common cause of cancer related death. Since the practice of PSA screening has become common the disease is most often found early and can have a long indolent course. Current definitive therapy treats the whole gland but has considerable long-term side effects. Focal therapies may be able to target the cancer while decreasing dose to organs at risk. Our objective was to determine if focal prostate brachytherapy could meet target objectives while permitting a decrease in dose to organs at risk in a way that would allow future salvage treatments. Further, we wanted to determine if focal treatment results in less toxicity. Utilizing the Medline repository, dosimetric papers comparing whole gland to partial gland brachytherapy and clinical papers that reported toxicity of focal brachytherapy were selected. A total of 9 dosimetric and 6 clinical papers met these inclusion criteria. Together, these manuscripts suggest that focal brachytherapy may be employed to decrease dose to organs at risk with decreased toxicity. Of current technology, image-guided HDR brachytherapy using MRI registered to transrectal ultrasound offers the flexibility and efficiency to achieve such focal treatments.

\section{Introduction}

Radiation therapy (RT) for prostate cancer came into common use in the United States utilizing an external beam approach during the 1920s. However, disease was often inadequately treated due to poor tissue penetration along with significant dermal morbidity produced by the treatment [1]. Combined with the development of androgen therapy there was lost interest in RT. Additionally, there was a decrease utilization of brachytherapy for all disease sites in the first half of the 20th century due to the significant radiation exposure to nurses and physicians. These shortcomings were overcome by the development of low activity radioactive iodine seeds that required minimal shielding during handling and, once placed in patients, released a negligible dose to the outside environment [2]. These seeds were first successfully employed for prostate cancer at Memorial Sloan Kettering in 1967, with hundreds of subsequent cases in the 1970s using the Whitmore open hand technique. Briefly, a laparotomy was performed and the prostate was mobilized from all but the posterior rectal facing wall. Evenly spaced empty catheters were then placed through the exposed prostate until felt by the physician rectally, followed by permanent seed placement. It was determined that doses greater than 100 Gy produced a five-year disease-free survival rate of $80 \%$. However, there were considerable long-term issues with inadequate coverage and morbidity from the invasive nature of the procedure; thus the technique was abandoned by the mid 1980s.

At the time that the free hand technique was in decline there were several advancements made in diagnostic imaging that would have profound impact on prostate brachytherapy. Holm was able to combine the application of ultrasound (US) guided seed placement for pancreatic cancer with transrectal US (TRUS) to develop US guided low dose rate (LDR) prostate brachytherapy. In Europe this was combined with external beam radiation therapy (EBRT) on a series of patients. However the high dose (160 Gy LDR and $46 \mathrm{~Gy}$ EBRT) resulted in significant side effects [3]. In the early 
1990s this technique was refined by utilizing lower doses, achieving outcomes similar to prostatectomy with less overall side effects [4]. By the mid 1990s high dose rate brachytherapy (HDR) was practiced at a handful of centers, which offered an additional degree of conformity by being able to shape the delivered dose based on computer optimization of source dwell times. As a result of these developments prostate brachytherapy either as monotherapy or as part of a combined EBRT regimen has become a mainstream therapeutic option for low risk and intermediate risk prostate cancer [5].

There are significant risks of side effects associated with either surgery or RT, including urinary toxicity, erectile dysfunction, and, in the case of RT, rectal toxicity. In the pre-PSA era, prostate disease was not detected until it was more advanced, requiring treatment of the entire gland and immediate periphery to control disease and impart increased survival. Thus such symptoms were viewed as an acceptable necessity. With the advent of PSA screening in the 1990s and a better understanding of the behavior of prostate cancer, disease in the modern PSA era is found significantly earlier and typically has a more indolent course [6]. In patients with low risk disease active surveillance is an acceptable option, as well as prostatectomy or brachytherapy +/- EBRT [5]. However, for younger patients with isolated lesions a more ideal treatment would be to treat the focus of disease without affecting the urethra or neurovascular bundles which cause the most detrimental side effects of traditional therapies, namely, genitourinary toxicity and erectile dysfunction (ED). These treatment related issues become increasingly significant as the average life span of the population increases, with future patients living increasingly longer with whatever side effects treatment imparts. With advances in imaging modalities partial prostate brachytherapy may be applied with limited toxicity to what is a large patient population who want a temporary measure, with low side effects, but still affords salvage RT or surgery in the future if needed.

In this paper, we perform a systematic review to explore the up-to-date dosimetric and clinical studies of focal and focused prostate brachytherapy. We postulate that focal brachytherapy may offer a salvageable therapy that provides lower side effects. Given the dosimetric advantages of HDR prostate brachytherapy $[7,8]$ and the expanded use of prostate/pelvic MRI in clinical workups, intraoperative planned HDR brachytherapy utilizing TRUS registered, MRI tumor delineation would provide a reliable and efficient strategy to implant focal prostate brachytherapy at most radiotherapy centers.

\section{Methods}

2.1. Selection of Studies. Two separate searches were performed with two slightly different search criteria. A PubMed database search for the terms "focal + brachytherapy + prostate" and for "partial + brachytherapy + prostate" with no limits placed on publication date was completed (10/16/2015). All non-English language manuscripts were then removed at which point the abstracts of the remaining manuscripts were read. Those manuscripts that did not concentrate on focal or focused prostate brachytherapy or were review papers were removed from this pool. Focused treatments where deemed as those in which the whole gland was treated with brachytherapy; however there was a focal boost in the plan in an attempt to deliver more dose to the gross tumor volume (GTV) and/or decrease toxicity to nearby organs at risk (OARs). Inclusion criteria for manuscripts in the final analysis included dosimetric studies that demonstrated treatment and organ at risk dosimetric data of whole gland (HG) compared to focal or focused treatments. Clinical study inclusion criteria included works involving focal or focused brachytherapy that reported treatment toxicity.

2.2. Data Extraction and Clinical Endpoints. Data extraction was conducted according to the Preferred Reporting Items for Systematic Reviews and Meta-Analyses (PRISMA) statement [9]. For each study, the following information was extracted: name of the first author, year of publication, number of participants included in analysis, brachytherapy type (HDR versus LDR), RT protocol (whether focal or focused and whether monotherapy or with EBRT), planning imaging modality, and treatment imaging modality. The primary measure of interest for the analysis of dosimetric studies was if OAR and GTV doses were reported for both partial and whole gland treatments which would provide evidence as to whether partial gland therapy is amenable to future definitive salvage therapy (what will be referred to as salvageability). The primary measure of interest for the analysis of clinical studies was toxicity, with secondary interests, if available, in treatment efficacy in regard to survival parameters and treatment dosimetry details. Preliminary analysis indicated that the data provided was insufficient for meta-analysis, thus this work was followed as a PRISMA style systematic review.

\section{Results}

3.1. Selection of Manuscripts. As outlined in Figure 1, the initial search yielded combined 154 results. Removal of duplicates and non-English language manuscripts reduced this number to 138. All review articles were removed which reduced the pool of papers to 99 . The abstracts of these manuscripts were then read to determine if the work concentrated on focal or focused prostate brachytherapy. Resulting 32 papers met these eligibility criteria and were read in entirety for final inclusion. Criteria for dosimetric papers considered for final inclusion were those in which disease target prescription and dose to OARs were characterized between whole gland and focal/focused treatments. Clinical papers were required to demonstrate side effects of focal/focused brachytherapy treatments. In total, 9 dosimetric manuscripts (Table 1) and 6 clinical manuscripts (Table 2) met all eligibility requirements.

3.2. Patterns between Selected Dosimetric Manuscripts. The nine dosimetric studies included in this systematic review were published between 2009 and 2015 with a heavy weighting towards more recent publications. There were three different groups/collaborators that contributed more than 


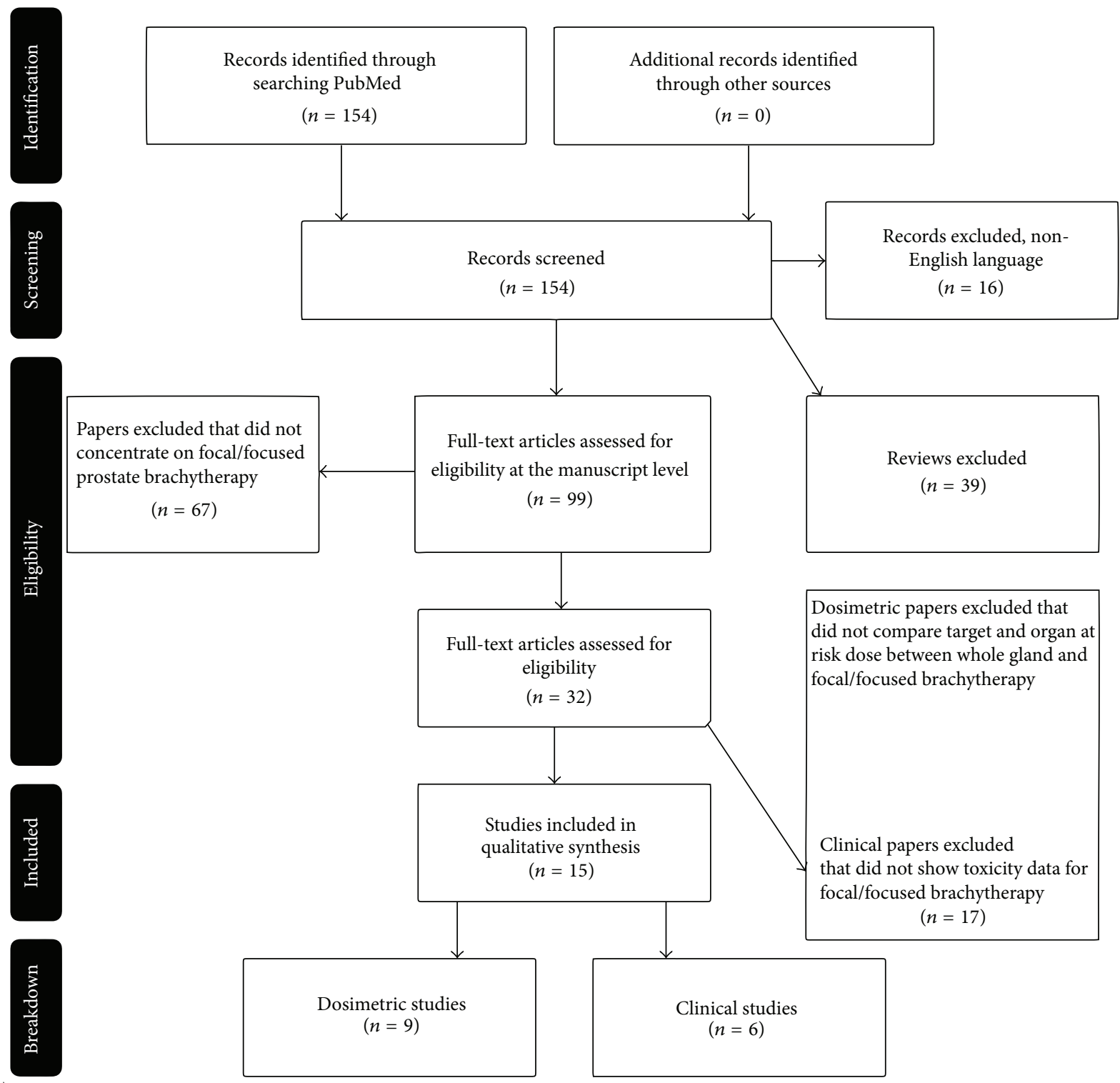

FIGURE 1: Literature selection process (PRISMA flow diagram).

one publication but looked at different aspects of focal and focused brachytherapy. These included significant work by Mason et al. and Al-Qaisieh et al. [10-13], Kamrava et al. and Banerjee et al. $[14,15]$, and Peters et al. and Moman et al. $[16,17]$. Sample size ranged from 2 to 15 with an average of 9 subjects per study. Of the manuscripts, 3 involved focused treatments [11, 13, 18], one hemigland treatment [14], two focal treatments $[17,19]$, and three hemi/focal treatments $[10,12,13]$, all of which were compared to whole gland therapy (Table 1). One work involved focal treatment as salvage therapy [17], while the rest were initial cancer treatment studies. In regard to planning imaging two manuscripts utilized CT to treat the whole prostate and an arbitrary focus $[14,15]$. The remaining works used various MRI sequences alone [11-13, 19], MRI with MR spectroscopy (MRS) [18], or MRI combined with positional information from prostate biopsy $[10,17]$. HDR was employed in 6 of the 9 studies [11$15,17]$ with LDR seeds in the rest $[10,18,19]$. Two of the HDR studies were plans combined with EBRT [11, 13], while the rest were brachytherapy monotherapy. In regard to HDR monotherapy fractionation, two studies employed a single fraction [12] (one of which was a salvage treatment [17]), while the rest used 6 fractions $[14,15]$.

Comparison of the target/OAR doses of the partial gland plans to whole gland therapy demonstrated three patterns. Two works using partial gland therapy demonstrated a similar dose to tumor volume and less dose to OARs $[14,19]$. Higher dose to the GTV was achieved by partial therapy while maintaining the same dose to the OARs as whole gland in two publications $[11,13]$. The remaining 5 manuscripts were able to achieve superior tumor dose with less involvement of the OARs with partial therapy $[10,12,15,17,18]$. As previously 


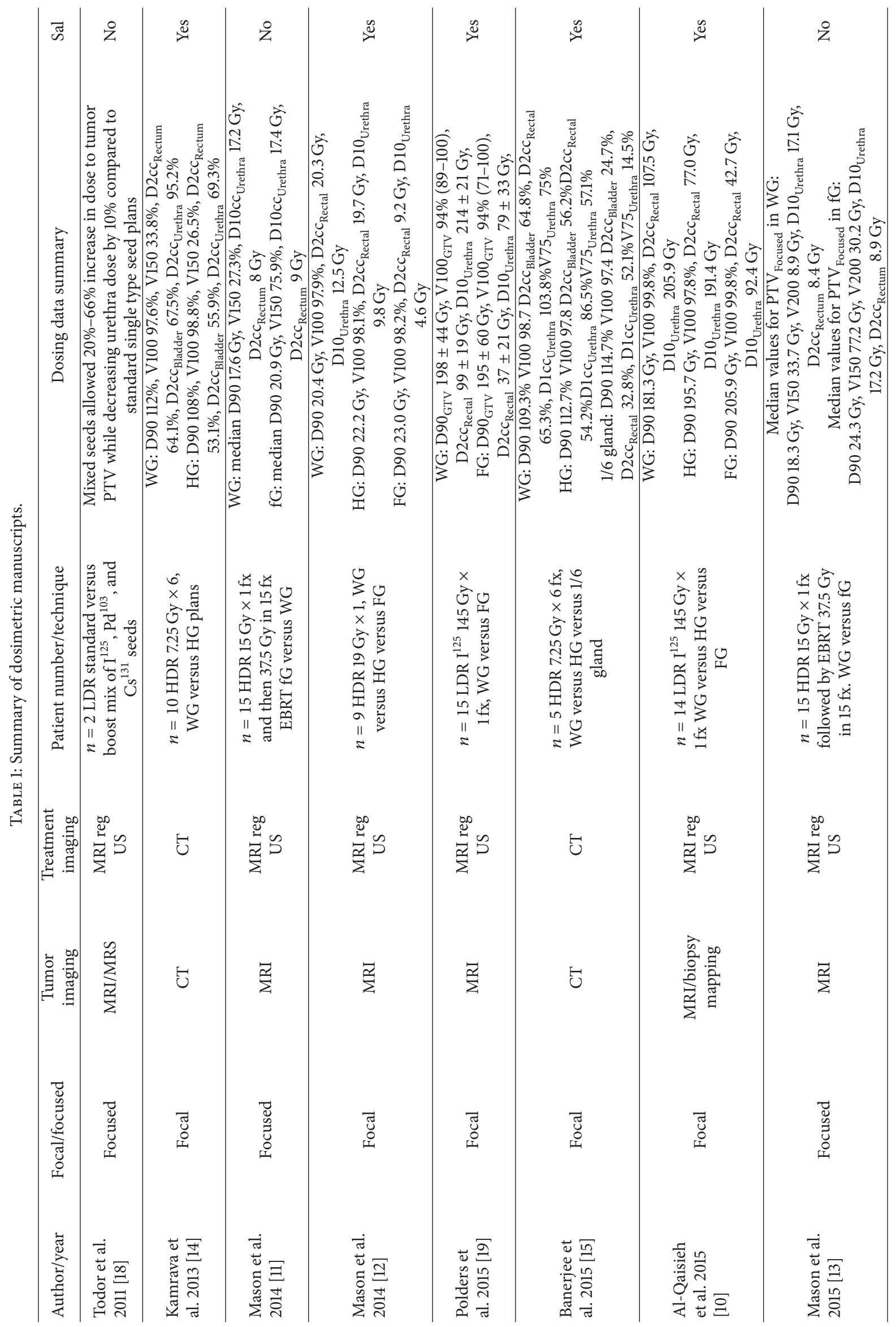




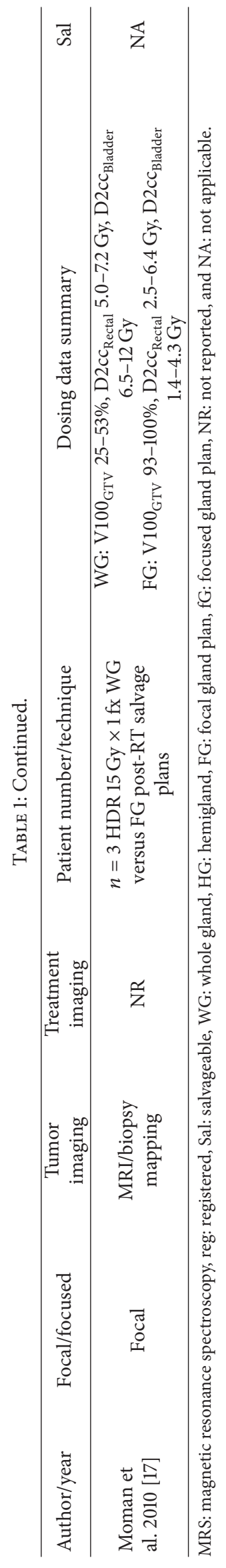




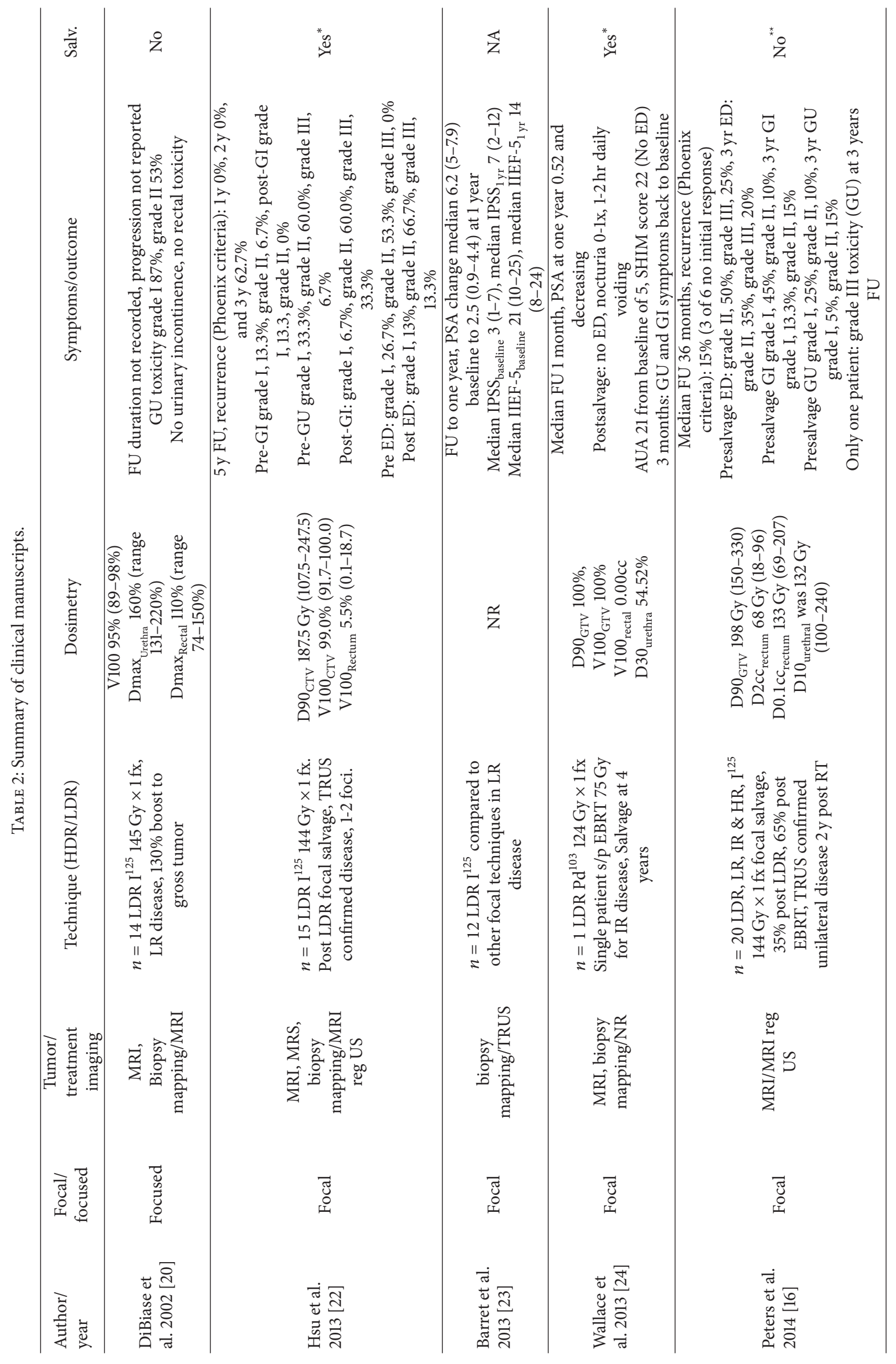




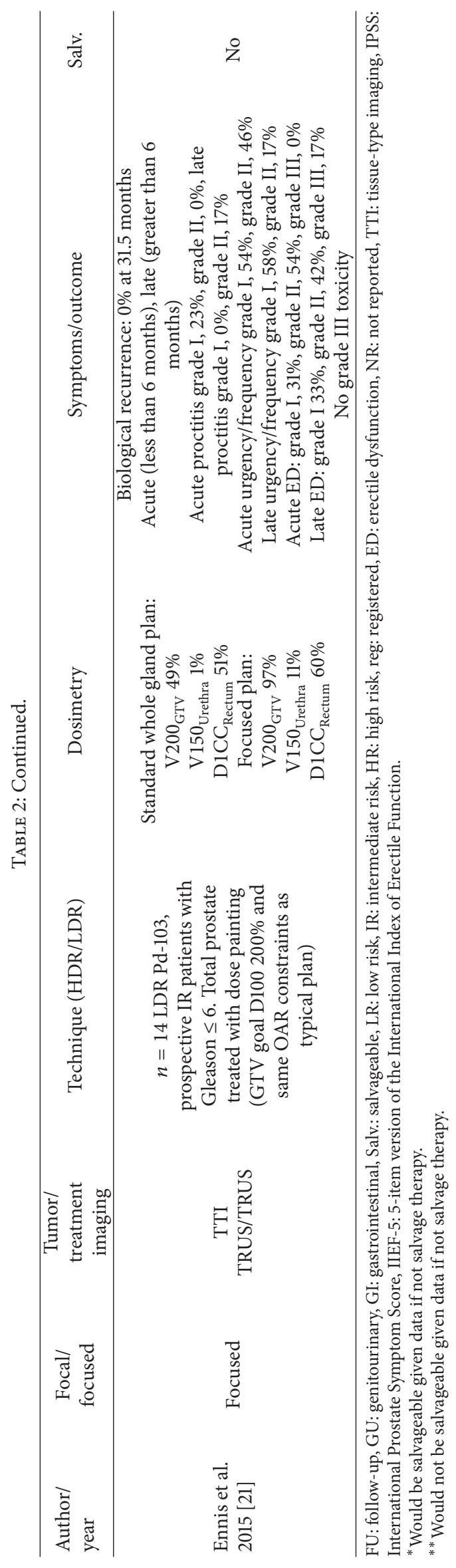


stated one major theoretical advantage of focal brachytherapy would be to permit definitive RT salvage in the future; therefore manuscripts were studied to determine salvageability. The authors in two of the publications demonstrated that hemi- and focal treatment plans $[14,15]$ would be amenable to future salvage. Based on typical OAR constraints three other studies $[10,12,19]$ could permit further definitive RT given the significant reduction in OAR dosing achieved from partial therapy.

3.3. Patterns between Selected Clinical Manuscripts. The six manuscripts that included clinical toxicity data of focal/ focused brachytherapy were published between 2002 and 2015 with all but one work completed on or after 2013. The number of patients assessed in the manuscripts ranged from 1 to 20 with a mean number of 7 subjects. Two of the six works utilized a focused $[20,21]$ rather than focal strategy $[16,22-24]$ (Table 2 ). The works were evenly divided between salvage treatments $[16,22,24]$ and initial treatments $[20,21$, 23 ] with two focused manuscripts being salvage treatments. Planning was based on MRI imaging [16], MRI combined with biopsy data [20,24], prostate biopsy mapping [23], and MRI combined with both MRS and biopsy data [22]. Treatment imaging utilized MRI registered TRUS [16, 22], MRI [20], and biopsy mapping with TRUS [21]. One manuscript utilized intraoperative planning and treatment guidance with tissue-type imaging (TTI) based TRUS that enables delineation of tumor volumes from US spectra [23]. Wallace et al. did not specify the method of treatment imaging [24]. All of the works employed LDR brachytherapy.

Dosimetric data was compared to consensus prostate brachytherapy constraints [25] to determine if the focal/ focused plan was able to increase tumor dose and decrease dose to OARs. All but one clinical work [23] included in this review published dosimetric data. Both focused works were able to achieve OAR doses typical of a standard whole gland treatment, with increased dose to the GTV [20, 21]. Ennis et al. provided examples of what the dosimetry would have been had they employed whole gland rather than focused plans [21]. The focused plans did slightly increase GTV dose than what would have been accomplished in the whole gland plan. Similarly, Peters et al. with focal brachytherapy were able to achieve higher than standard whole gland prostate GTV dosing while remaining within expected OAR tolerances [16]. The two remaining focal manuscripts were able to achieve significantly lower doses to the OARs, while delivering a dose to the target tumor that is comparable to [24] or greater [22] than what would be achieved with whole gland therapy.

Follow-up ranged from 1 to 60 months with a mean of 23 months. Follow-up duration was not published by DiBiase et al., nor was any disease progression information included [20]. There were no recurrences/failures in three manuscripts with median follow-up of 1 month [24], 12 months [23], and 31.5 months [21]. Hsu et al. had a 5-year biological failure rate as determined by Phoenix criteria of $28.6 \%$ [22]. Similarly, Peters et al. demonstrated failure via Phoenix criteria of $15 \%$ at 36 months, although half of those were patients that did not respond to therapy initially in regard to PSA [16]. When examining the selected publications' toxicity, the overall reported side effects are no more than would be expected for whole gland brachytherapy [26]. Closer examination of focal plans that significantly reduced OARs demonstrated patterns of decreased toxicity. In particular Hsu et al. with an average $\mathrm{V} 100_{\text {rectum }}$ of $5.5 \%$ showed only grade I GI toxicity in $13 \%$ of the patients [22], while the single subject in the report of Wallace et al. had no long-term side effects at 3 months in the background of significantly reduced rectal and urethra dose [24]. ED was not significantly changed from pre-RT levels in most studies $[16,20,23,24]$ although two had an increase in the proportion of ED being grade III versus grade I/II from the period before to that after treatment [21, 22], with one work achieving otherwise low side effects and low OAR dose [22].

\section{Discussion}

The dosimetric portion of the studies selected in this systematic review demonstrate that a focal plan is attainable in regard to both GTV dosing and decreasing dose to OARs using either LDR or HDR approaches. Furthermore, there is evidence that such partial gland treatments may permit irradiation of prostate tissue outside the GTV for future definitive, salvage RT $[14,15]$. Although the number of clinical studies and the number of treated patients are low, focal and focused plans were able to deliver lower than usual dose to OARs with excellent outcomes from a toxicity standpoint. For example, Ennis et al. were able to achieve a significant reduction to the rectal dose that resulted in less GI toxicity than would have been anticipated by a whole gland treatment [22]. Similar to the dosimetric works, the two focal clinical studies with decreased OAR dose would likely be amenable to future salvage RT or surgery [22, 24]. In our own patient population we have found that select low risk patients are appropriate for focal HDR treatments. Figures 2(a), 2(b), and 2(e) demonstrated an example patient where simulation of focal therapy was able to achieve target dose with minimal exposure to OARs and afford future salvage therapy within OAR and target dose constraints. The ultimate composite between the focal and salvage plan is comparable to standard whole gland therapy (Figures 2(c), 2(d), and 2(e) and Table 3). Based on these results and our own experience, the authors of this work feel that sufficient evidence exists to pursue larger clinical studies of focal therapy for well-selected low risk prostate cancer patients.

In the PSA era there is significant lead-time with the detection of early stage disease [6]. For those patients who have extended life expectancy and low risk disease current options are limited to active surveillance, prostatectomy, or definitive RT [5]. The side effects of both whole gland RT and surgery, including the $50 \%$ reported incidence of ED [27], dissuade many patients from therapy who may otherwise be active and healthy. On the other hand there are many patients for whom active surveillance provokes too much anxiety, not to mention the morbidity that potential annual biopsy can induce. Essentially, these patients desire a treatment to halt disease progression with low toxicity. The evidence presented in this manuscript suggests that focal brachytherapy can be 


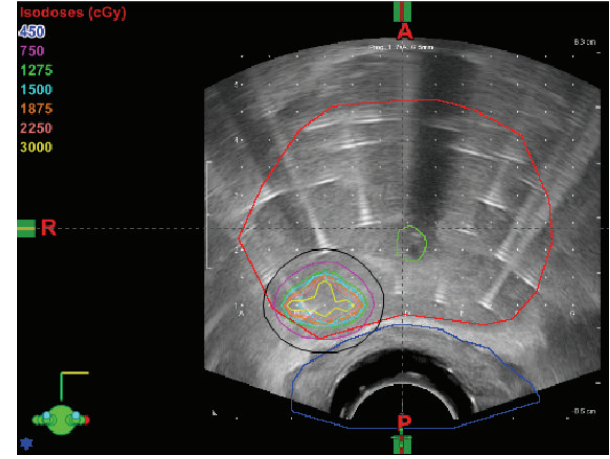

Relative dose (\%)

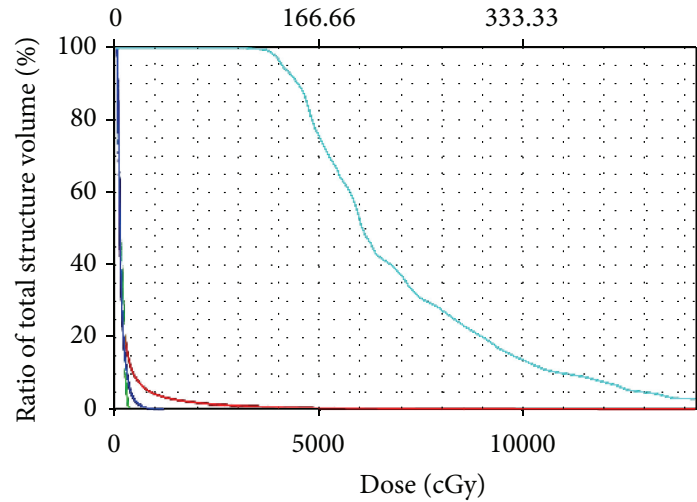

(a)

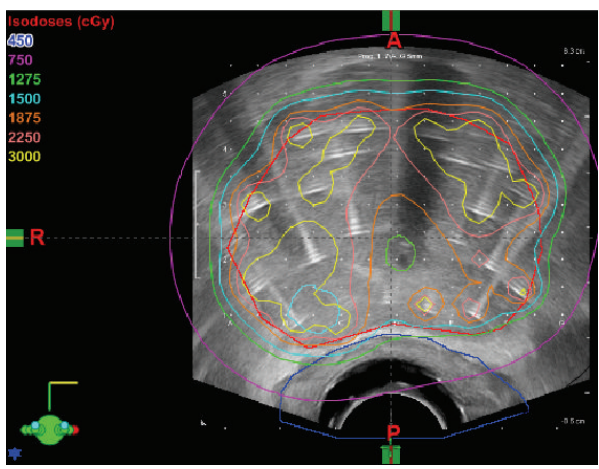

Relative dose (\%)

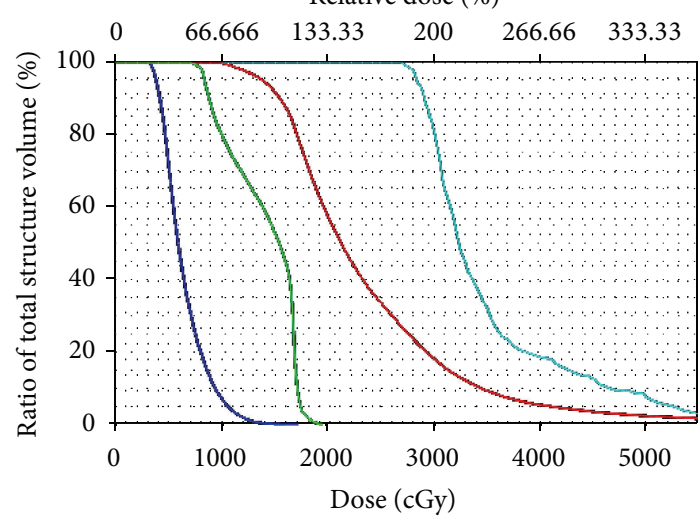

(c)

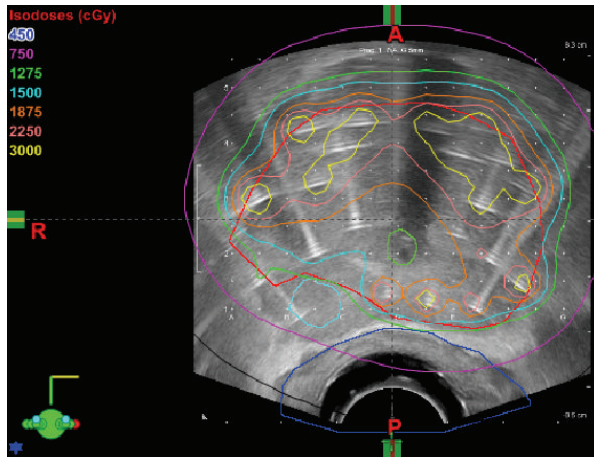

Relative dose (\%)

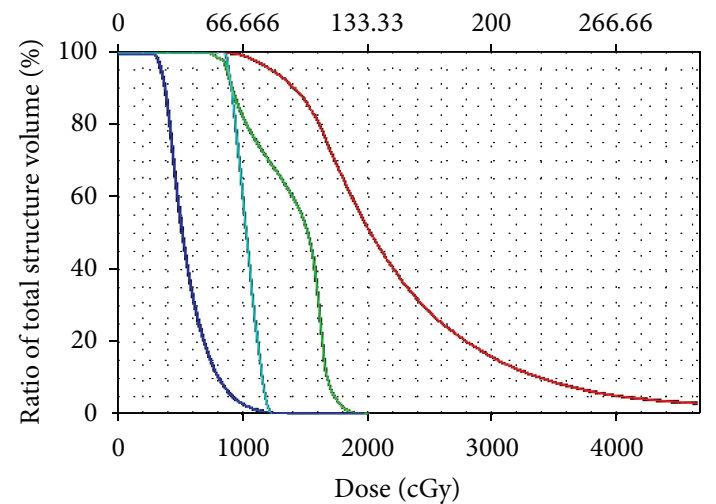

(b)

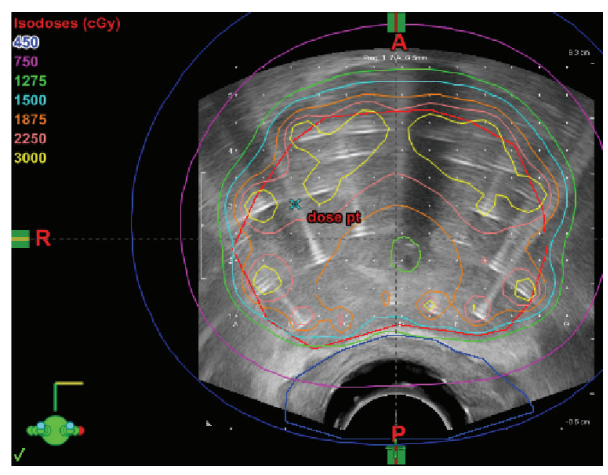

Relative dose (\%)

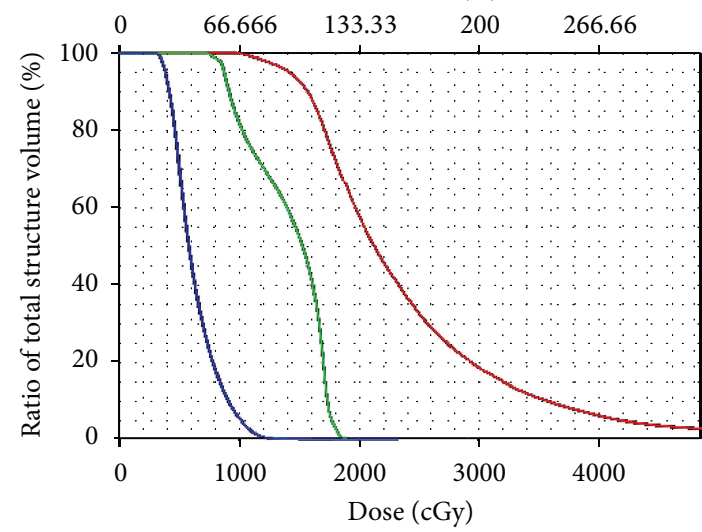

(d)

Figure 2: Continued. 


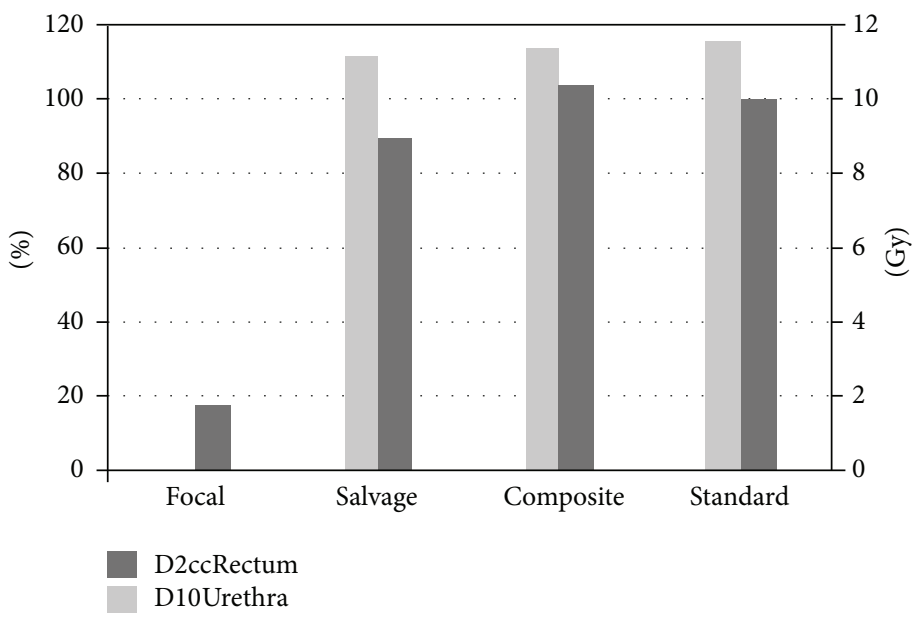

(e)

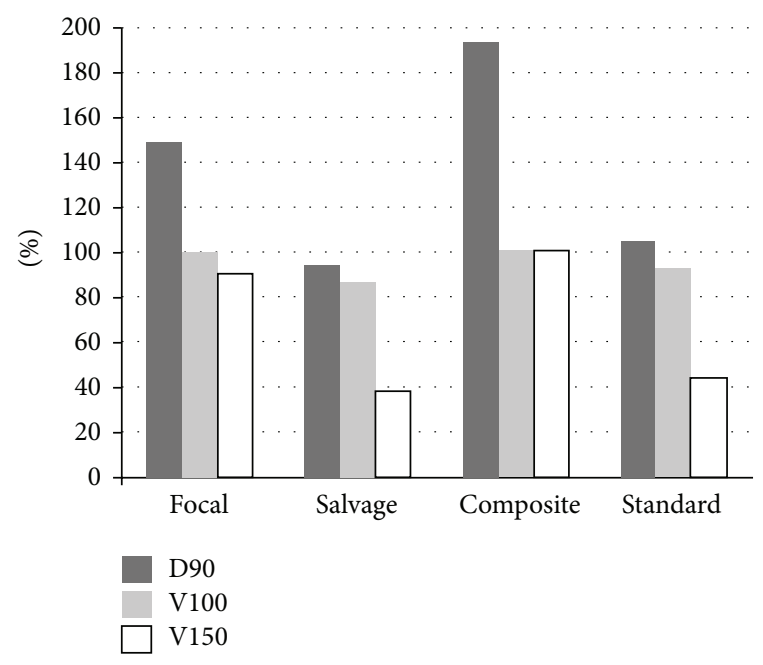

(f)

FIGURE 2: Organ/isodose contours, DVH for focal plan (a), salvage plan (b), composite of focal plan and salvage plan (c), and standard whole gland plan (d) of ideal focal HDR patient. Isodose lines are as follows: Black 450 cGy, Magenta 750 cGy, Green 1275 cGy, Cyan 1500 cGy, Orange 1875 cGy, Dark Pink 2250 cGy, and Yellow 3000 cGy. Target (e) and organ at risk doses (f).

TABLE 3: Experimental dose to target and OAR between focal, salvage, and whole gland HDR brachytherapy.

\begin{tabular}{|c|c|c|c|c|}
\hline Parameter & Focal & Salvage & Composite & Standard \\
\hline $\mathrm{D} 2 \mathrm{cc}_{\text {Rectum }}(\mathrm{Gy})$ & 1.76 & 8.90 & 10.29 & 9.98 \\
\hline $\mathrm{D} 10_{\text {Urethra }}(\%)$ & 0.00 & 111.66 & 113.85 & 115.64 \\
\hline D90 ${ }_{\text {PTV }}(\%)$ & 149.30 & 94.15 & 193.9 & 104.54 \\
\hline V100 ${ }_{\text {PTV }}(\%)$ & 100.00 & 86.51 & 100.00 & 92.70 \\
\hline $\mathrm{V} 150_{\mathrm{PTV}}(\%)$ & 89.70 & 37.89 & 100.00 & 43.72 \\
\hline
\end{tabular}

optimized in low risk patients to deliver a fully therapeutic dose to the GTV while avoiding significant side effects. Further this treatment may possibly be salvageable with either surgery or RT with future disease recurrence. While current definitive treatment with surgery or whole gland RT can be technically salvaged, there are issues with both. After whole prostate RT salvage prostatectomy is difficult to perform on what is an extremely fibrotic post-RT prostate and surrounding tissues [28]. Alternatively, salvage RT is more often treating a significantly greater portion of the pelvis with greater side effects than initial whole gland therapy. Focal brachytherapy may be a more salvageable option that could act as a temporizing bridge to definitive therapy. In doing so, focal brachytherapy could be used to extend a period that is free of morbidity before definitive surgery/RT or in the bestcase scenario negate any further treatment.

While none of the clinical papers employed HDR brachytherapy, the technique offers theoretical advantages over LDR for focal treatments [7]. First, the treatment volume can be optimized through inverse planning, which allows compensation for suboptimal needle placement and an overall more reliable dose distribution pattern in regard to the PTV [29]. There are fewer radiation concerns in regard to exposure to physician, nurses, and physicists as the source is delivered remotely. Also, there is no need for postimplant dosimetry, which adds to an already efficient planning/treatment delivery system. With the goal to create a salvageable focal plan to spare OAR and maximize dose to the GTV, the flexibility offered by HDR is more optimal. One significant drawback to HDR as monotherapy is that it is traditionally fractionated, which requires multiple needle insertions, or keeping needles placed for prolonged period of times. However, there is some experience with single fraction HDR [30], which likely would suffice for focal therapy given the dosimetric work of Kamrava et al. and Moman et al. [14, 17].

An additional benefit of HDR over LDR is its efficient integration with TRUS guidance, allowing a scan-plan-treat environment [8]. TRUS provides improved visualization of the base and apex of the prostate compared to CT and eliminates the need to move the patient from an imaging room to a brachytherapy suite, saving considerable procedure time. However, one significant drawback is that tumors are not readily visualized and as an imaging modality it takes experience to read. There is (TTI) TRUS which has shown success in delineating tumor from normal prostate [21]; however, the imaging requires some skill with interpretation and as a technique is not in wide use. Alternatively, tumors are readily visualized by MRI. However, the long acquisition time and usually off-site location of MRI scanners preclude them from streamlined HDR simulation, planning, and delivery except at a few specialized centers [20]. As shown by many of the manuscripts selected in this review prior pelvic MRI can be registered to the treatment TRUS, which when combined provides efficient intraprocedural tumor delineation. In regard to focal therapy, Mason et al. confirmed that MRI with T2-weighted, diffusion-weighted, and dynamiccontrast-enhanced sequences can accurately detect tumors, negating biopsy mapping [11]. To combine all of the above technologies together, treatment planning systems such as 


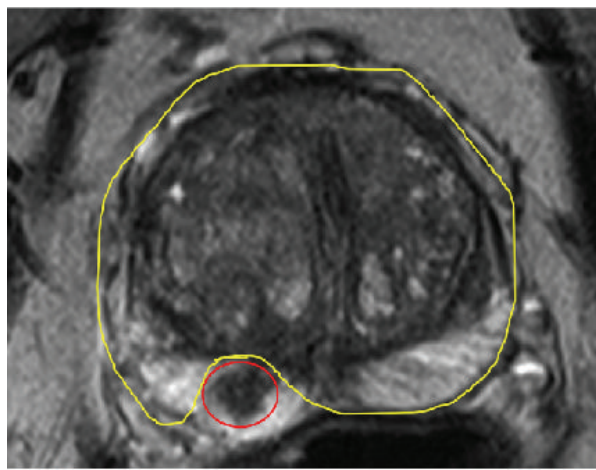

(a)

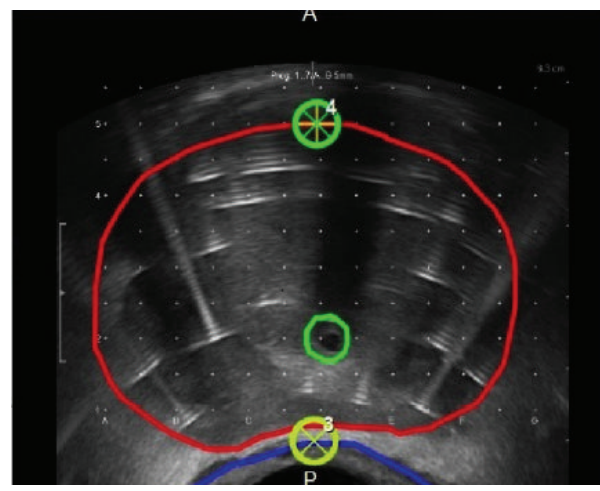

(c)

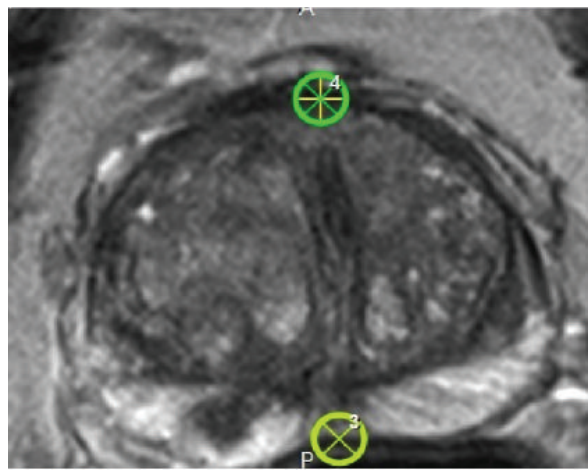

(b)

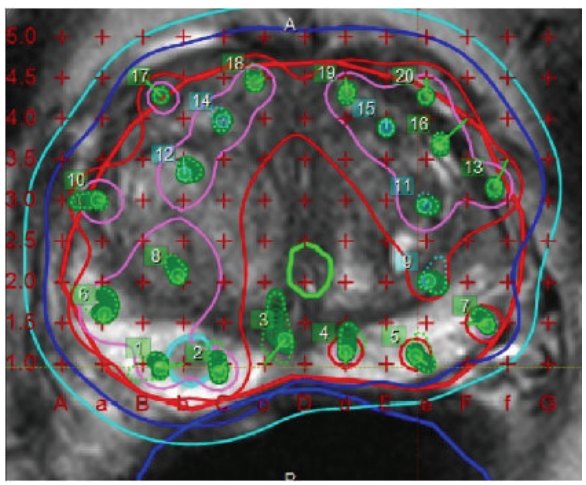

(d)

FIGURE 3: (a) Preprocedural MRI demonstrating GTV (yellow) and nondiseased prostate (red). Preprocedural MRI using two anchor points (green and yellow circles) (b) are able to be registered to intraprocedural TRUS with $2.5 \mathrm{~mm}$ grid spacing (c). (d) The resulting merge of the TRUS grid and preprocedural MRI with GTV (thick light blue), urethra (thick solid light green), rectum (thick blue line), and isodose lines (thin lines) for typical whole gland plan using $18 \mathrm{HDR}$ catheters.

Vitesse Version 3.0 (Varian, Inc. Palo Alto, CA) that supports HDR intraprocedural TRUS guided needle placement, planning, and delivery already exist which as a software can be readily registered with prior MRI data. This registration has been readily performed experimentally at our own institution as shown in Figure 3. Given the increased utilization of pelvic MRI in prostate cancer workup, MRI registered TRUS is an efficient and reliable focal brachytherapy platform that can be adapted to most brachytherapy centers.

There are areas that, based on this systematic review, require further research to validate focal HDR prostate brachytherapy as a reliable technique. Foremost, the current clinical trials are few in number, have short follow-up duration, and are small in sample size. Therefore, it is a plausible that the clinical results presented in this systematic review may not be accurate representations of the toxicity and efficacy from focal plans. Further, the disease stage varied amongst the papers including those with more advanced disease than low risk prostate cancer that would be more applicable to focal treatments. To the knowledge of the authors, there are also no works that directly compare whole gland to focal treatments in regard to patient toxicity and treatment efficacy. These shortcomings would be clarified with a trial of whole gland and focal brachytherapy that is large enough to permit a comparison of toxicity and efficacy, that is, progression free survival, with significant power. In regard to HDR as monotherapy, single fraction therapy would be more attractive to patients than multiple fractions with either prolonged needle placement or repeat needle application. However, there has been very limited publication experience with single fraction HDR monotherapy, thus requiring more research before application as a part of focal brachytherapy strategy.

\section{Conclusion}

In this systematic review we demonstrated dosimetrically that focal brachytherapy could be appropriately applied to prostate tumors while decreasing dose to OARs. Clinical studies were also presented that provided evidence that such treatments decreased toxicity over current whole gland therapy. The decreased toxicity and decreased dose to OARs permit potential salvage RT or salvage prostatectomy if there is future recurrence. Given the indolent nature of prostate cancer when caught at an early stage, focal therapy may serve as a temporary and possible permanent measure for low risk prostate cancer that delays/avoids the significant toxicity such as ED that current definitive therapy has. To this end, 
HDR brachytherapy allows the dosimetric flexibility required for focal plans. Further, pelvic MRIs, which are increasingly obtained in prostate cancer workup, can be registered to intraprocedural TRUS for an efficient and widely applicable focal brachytherapy platform.

\section{Competing Interests}

The authors declare that they have no competing interests.

\section{References}

[1] S. Sriprasad, M. R. Feneley, and P. M. Thompson, "History of prostate cancer treatment," Surgical Oncology, vol. 18, no. 3, pp. 185-191, 2009.

[2] J. N. Aronowitz, "Whitmore, Henschke, and Hilaris: the reorientation of prostate brachytherapy (1970-1987)," Brachytherapy, vol. 11, no. 2, pp. 157-162, 2012.

[3] H. H. Holm, "Interventional ultrasound in Europe," Ultrasound in Medicine \& Biology, vol. 24, no. 6, pp. 779-791, 1998.

[4] H. Ragde, G. L. Grado, B. Nadir, and A.-A. Elgamal, "Modern prostate brachytherapy," CA: A Cancer Journal for Clinicians, vol. 50, no. 6, pp. 380-393, 2000.

[5] National Comprehensive Cancer Network, Prostate Cancer (Version 1.2015), 2015.

[6] J. Hugosson, S. Carlsson, G. Aus et al., "Mortality results from the Göteborg randomised population-based prostate-cancer screening trial," The Lancet Oncology, vol. 11, no. 8, pp. 725-732, 2010.

[7] B. Libby, "Implementation of image-guided prostate HDR brachytherapy using MR-ultrasound fusion," in Proceedings of the 57th Annual Meeting of the AAPM, Anaheim, Calif, USA, July 2015, Medical Physics, vol. 42, no. 6, p. 3572, 2015.

[8] B. Libby, "Real time ultrasound guidance for optimizing high dose rate prostate brachytherapy," in Proceedings of the 55th Annual Meeting of the AAPM, Medical Physics 40:6, Invited oral presentation, Indianapolis, Ind, USA, August 2013.

[9] D. Moher, A. Liberati, J. Tetzlaff, and D. G. Altman, "Preferred reporting items for systematic reviews and meta-analyses: the PRISMA statement," Journal of Clinical Epidemiology, vol. 62, no. 10, pp. 1006-1012, 2009.

[10] B. Al-Qaisieh, J. Mason, P. Bownes et al., "Dosimetry modeling for focal low-dose-rate prostate brachytherapy," International Journal of Radiation Oncology, Biology, Physics, vol. 92, no. 4, pp. 787-793, 2015.

[11] J. Mason, B. Al-Qaisieh, P. Bownes et al., "Multi-parametric MRI-guided focal tumor boost using HDR prostate brachytherapy: a feasibility study," Brachytherapy, vol. 13, no. 2, pp. 137-145, 2014.

[12] J. Mason, B. Al-Qaisieh, P. Bownes, D. Thwaites, and A. Henry, "Dosimetry modeling for focal high-dose-rate prostate brachytherapy," Brachytherapy, vol. 13, no. 6, pp. 611-617, 2014.

[13] J. Mason, P. Bownes, B. Carey, and A. Henry, "Comparison of focal boost high dose rate prostate brachytherapy optimisation methods," Radiotherapy and Oncology, vol. 117, no. 3, pp. 521524, 2015.

[14] M. Kamrava, M. P. Chung, O. Kayode et al., "Focal high-doserate brachytherapy: a dosimetric comparison of hemigland vs. conventional whole-gland treatment," Brachytherapy, vol. 12, no. 5, pp. 434-441, 2013.
[15] R. Banerjee, S.-J. Park, E. Anderson, D. J. Demanes, J. Wang, and M. Kamrava, "From whole gland to hemigland to ultra-focal high-dose-rate prostate brachytherapy: a dosimetric analysis," Brachytherapy, vol. 14, no. 3, pp. 366-372, 2015.

[16] M. Peters, M. Maenhout, J. R. N. van der Voort Van Zyp et al., "Focal salvage iodine-125 brachytherapy for prostate cancer recurrences after primary radiotherapy: a retrospective study regarding toxicity, biochemical outcome and quality of life," Radiotherapy and Oncology, vol. 112, no. 1, pp. 77-82, 2014.

[17] M. R. Moman, C. A. T. van den Berg, A. E. Boeken Kruger et al., "Focal salvage guided by T2-weighted and dynamic contrast-enhanced magnetic resonance imaging for prostate cancer recurrences," International Journal of Radiation Oncology Biology Physics, vol. 76, no. 3, pp. 741-746, 2010.

[18] D. A. Todor, I. J. Barani, P.-S. Lin, and M. S. Anscher, "Moving toward focal therapy in prostate cancer: dual-isotope permanent seed implants as a possible solution," International Journal of Radiation Oncology Biology Physics, vol. 81, no. 1, pp. 297-304, 2011.

[19] D. L. Polders, M. van Herk, K. Nichol et al., "Establishing implantation uncertainties for focal brachytherapy with I-125 seeds for the treatment of localized prostate cancer," Acta Oncologica, vol. 54, no. 6, pp. 839-846, 2015.

[20] S. J. DiBiase, K. Hosseinzadeh, R. P. Gullapalli et al., "Magnetic resonance spectroscopic imaging-guided brachytherapy for localized prostate cancer," International Journal of Radiation Oncology Biology Physics, vol. 52, no. 2, pp. 429-438, 2002.

[21] R. D. Ennis, S. A. Quinn, F. Trichter et al., "Phase I/II prospective trial of cancer-specific imaging using ultrasound spectrum analysis tissue-type imaging to guide dose-painting prostate brachytherapy," Brachytherapy, vol. 14, no. 6, pp. 801-808, 2015.

[22] C. C. Hsu, H. Hsu, B. Pickett et al., "Feasibility of MR imaging/MR spectroscopy-planned focal partial salvage permanent prostate implant (PPI) for localized recurrence after initial PPI for prostate cancer," International Journal of Radiation Oncology, Biology, Physics, vol. 85, no. 2, pp. 370-377, 2013.

[23] E. Barret, Y. Ahallal, R. Sanchez-Salas et al., "Morbidity of focal therapy in the treatment of localized prostate cancer," European Urology, vol. 63, no. 4, pp. 618-622, 2013.

[24] T. Wallace, I. Avital, A. Stojadinovic, B. Brucher, E. Coté, and J. Yu, "Multi-parametric mri-directed focal salvage permanent interstitial brachytherapy for locally recurrent adenocarcinoma of the prostate: a novel approach," Journal of Cancer, vol. 4, no. 2, pp. 146-151, 2013.

[25] C. Salembier, P. Lavagnini, P. Nickers et al., "Tumour and target volumes in permanent prostate brachytherapy: a supplement to the ESTRO/EAU/EORTC recommendations on prostate brachytherapy," Radiotherapy and Oncology, vol. 83, no. 1, pp. 3-10, 2007.

[26] A. Henderson, R. W. Laing, and S. E. M. Langley, "Quality of life following treatment for early prostate cancer: Does Low Dose Rate (LDR) brachytherapy offer a better outcome? a review," European Urology, vol. 45, no. 2, pp. 134-141, 2004.

[27] A. L. Potosky, W. W. Davis, R. M. Hoffman et al., "Fiveyear outcomes after prostatectomy or radiotherapy for prostate cancer: the prostate cancer outcomes study," Journal of the National Cancer Institute, vol. 96, no. 18, pp. 1358-1367, 2004.

[28] E. Rogers, M. Ohori, V. S. Kassabian, T. M. Wheeler, and P. T. Scardino, "Salvage radical prostatectomy: outcome measured by serum prostate specific antigen levels," The Journal of Urology, vol. 153, no. 1, pp. 104-110, 1995. 
[29] D. J. Brenner and E. J. Hall, "Fractionation and protraction for radiotherapy of prostate carcinoma," International Journal of Radiation Oncology Biology Physics, vol. 43, no. 5, pp. 1095-1101, 1999.

[30] P. Hoskin, A. Rojas, P. Ostler et al., "High-dose-rate brachytherapy alone given as two or one fraction to patients for locally advanced prostate cancer: acute toxicity," Radiotherapy and Oncology, vol. 110, no. 2, pp. 268-271, 2014. 


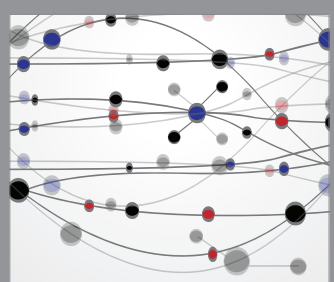

The Scientific World Journal
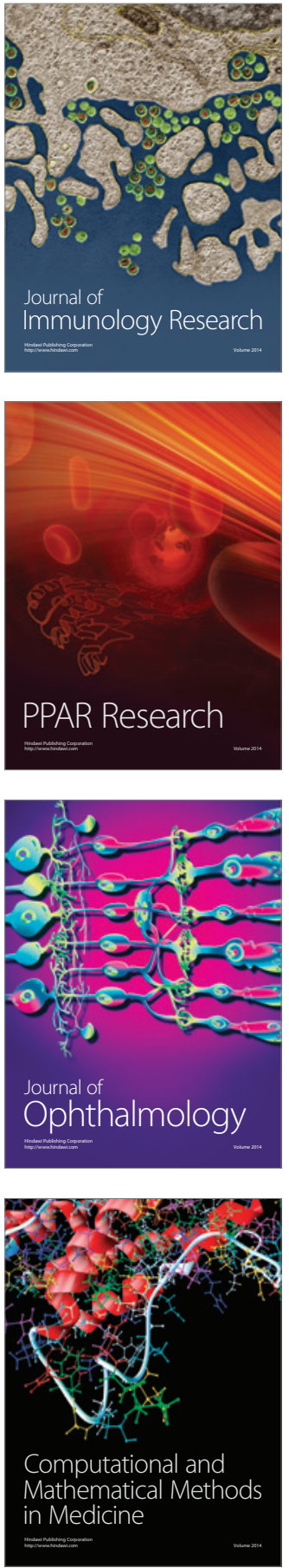

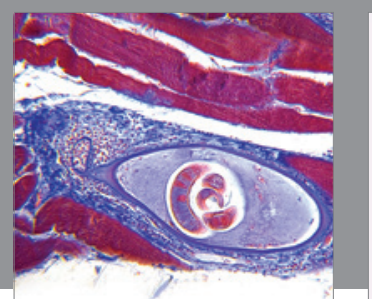

Gastroenterology Research and Practice

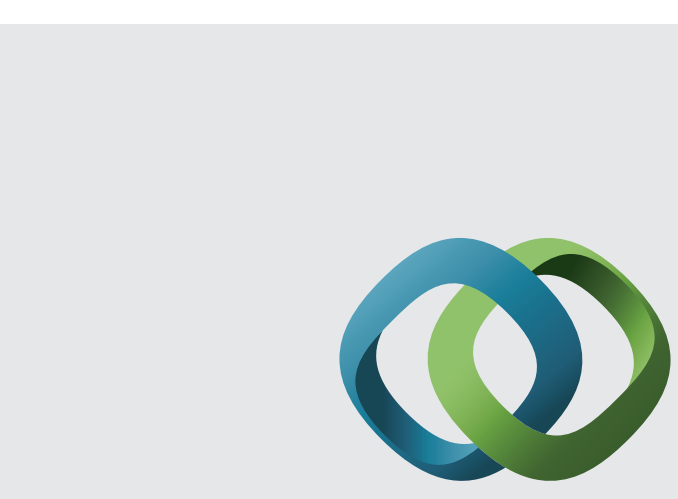

\section{Hindawi}

Submit your manuscripts at

http://www.hindawi.com
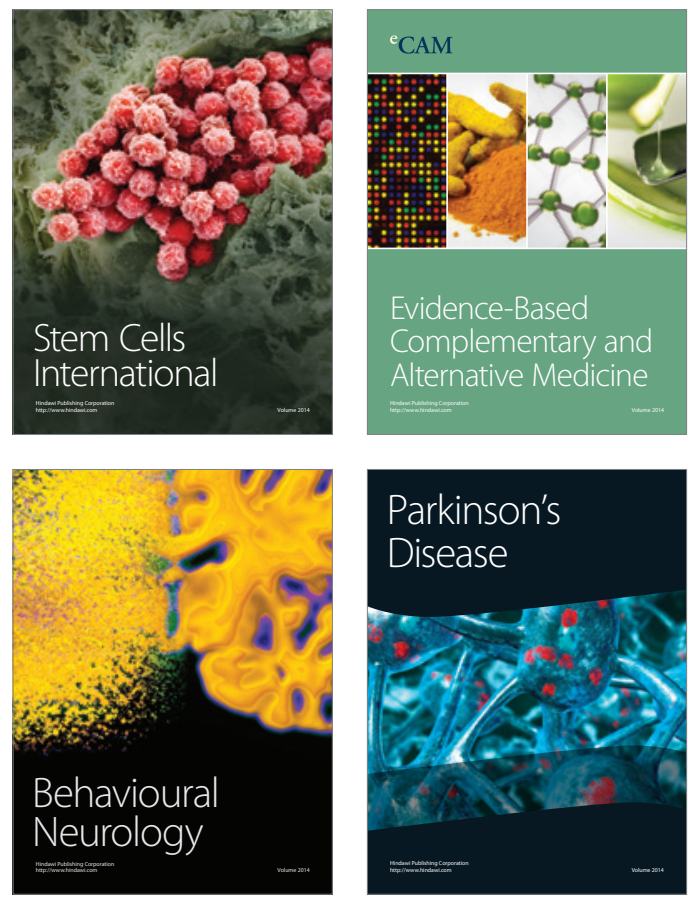
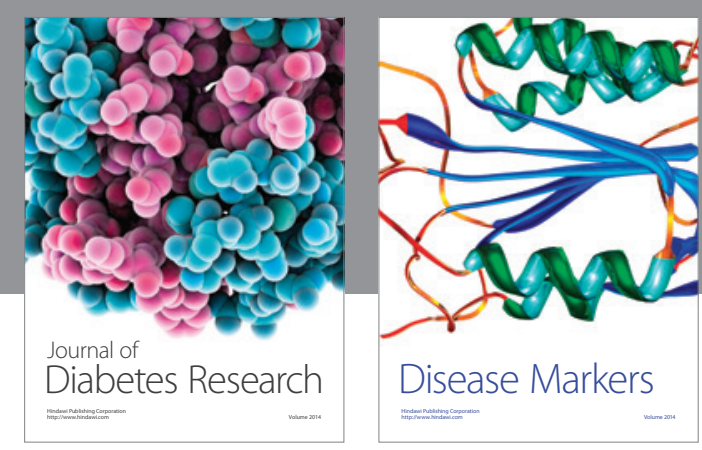

Disease Markers
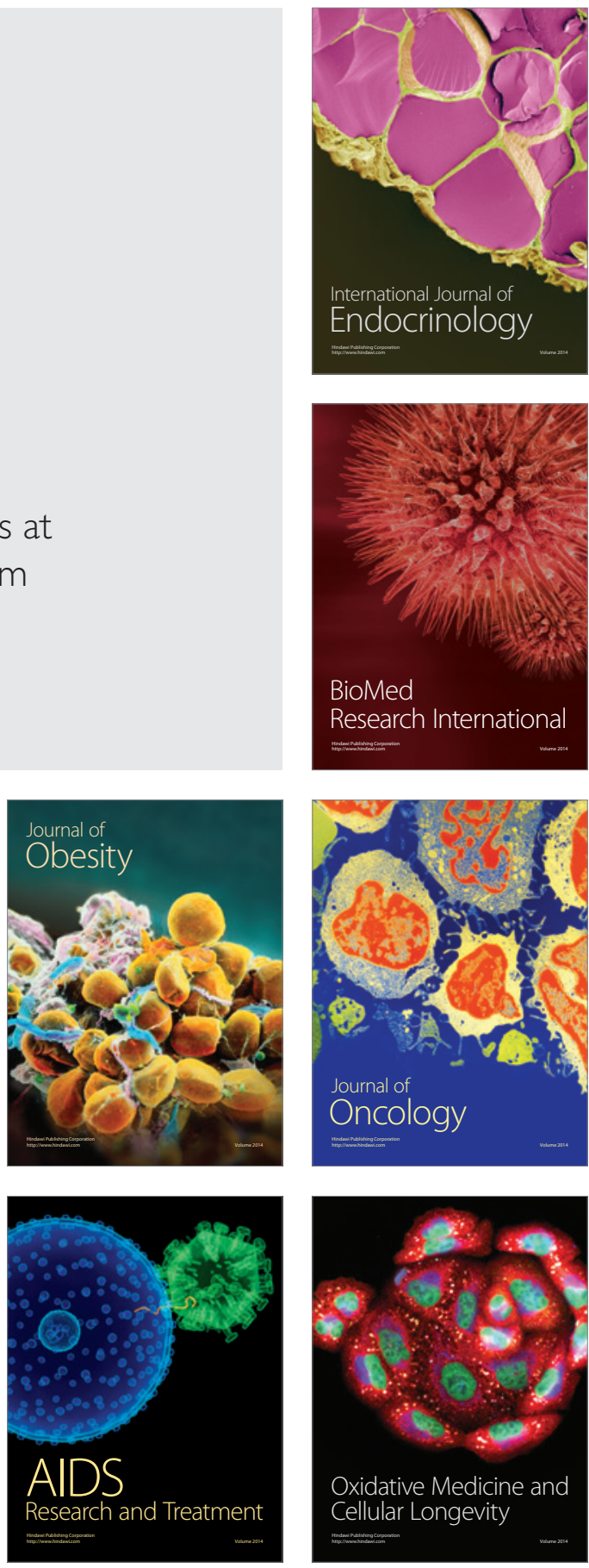